\title{
Solutions of generalized fractional perturbed Zakharov-Kuznetsov equation arising in a magnetized dusty plasma
}

\author{
L. Akinyemi \\ Department of Mathematics, Lafayette College, Easton, Pennsylvania, USA. \\ e-mail: akinyeml@lafayette.edu
}

Received 16 December 2020; accepted 23 February 2021

\begin{abstract}
The generalized fractional perturbed $(3+1)$-dimensional Zakharov-Kuznetsov (PZK) equation, which appears in the magnetized two-iontemperature dusty plasma, and quantum physics is considered. The sub-equation method in the conformable sense is proposed to obtain exact solutions to this equation. The new solutions obtained by the proposed method are dark soliton, multi-soliton, solitary wave, kink-shape, bell-shaped soliton, and periodic solutions that are substantial in the field of mathematical physics and can be of relevance in the field of plasma physics, also for future research.
\end{abstract}

Keywords: Perturbed $(3+1)$-dimensional Zakharov-Kuznetsov equation; conformable derivative; sub-equation method; Riccati equation; mathematical physics.

PACS: 02.30.Jr; 47.35.Fg; 52.35.FP; 52.35.MW; 52.35.Sb

DOI: https://doi.org/10.31349/RevMexFis.67.060703

\section{Introduction}

The nonlinear evolution equations have played a fundamental role in the field of mathematical physics and many other areas of applied science. As an instance, in signal processing, dusty plasma, fluid dynamics, nonlinear optics, quantum mechanics, biology, and so forth [1-6]. Examining the exact solutions of these nonlinear models help us to understand the mechanism, application and gives better knowledge of the model. With the virtue of these solutions, a better vision can be captured into the physical feature of the considered model. In recent years, series of methods have been developed to obtain the exact and approximate solutions of the nonlinear evolution equations in mathematical physics, as an instance, the Jacobi elliptic function method [7], Adomian decomposition method [8], sine-cosine method $[9,10]$, first integral method $[11,12]$, variational iteration method $[13,14]$, extended tanh method $[15,16]$, qhomotopy analysis method [17-19], exp-function method [20-22], q-homotopy analysis transform method [23-25], tanh-sech method [26, 27], homotopy perturbation method [28], $\left(G^{\prime} / G\right)$-expansion method [29,30], fractional reduced differential transform method $[31,32]$, homogeneous balance method [33,34], inverse scattering method [35], iterative shehu transform method [36], homotopy analysis method [37, 38], Jacobi elliptic expansion method [39, 40], residual power series method [41-43], perturbation-iteration algorithm [44, 45], modified Kudryashov method [46], new extended direct algebraic method [47], Sardar sub-equation method [48], Sine-cosine and sinh-cosh techniques [49], simple equation method [50] and so on.

The $(3+1)$-dimensional Zakharov-Kuznetsov (ZK) equation which comprises of the nonlinear term " $P P_{x}$ " and thirdorder dispersion term " $P_{x x x}$ " is restricted to the waves of small amplitudes only is presented by

$$
\Delta:=P_{t}+A P P_{x}+B P_{x x x}+C\left(P_{x y y}+P_{x z z}\right)=0,
$$

where $P$ represents electrostatic potential, the physical quantities $A, B$, and $C$ are constants. Seadawy et al. [51,52] and Zhen et al. [53] have outlined these physical quantities. The width of the soliton and its velocity deviate from the predictions of this equation when the amplitude of the wave increases. As a result, an additional fifth-order dispersion term which is a higher-order dispersion term, "E $P_{x x x x x}$ ", is added to Eq. (1) to overcome this problem (see [51-54], for more detailed). The new perturbed $(3+1)$-dimensional ZK equation reads

$$
\begin{aligned}
\Delta: & =P_{t}+A P P_{x}+B P_{x x x} \\
& +C\left(P_{x y y}+P_{x z z}\right)+\mathcal{E} P_{x x x x x}=0 .
\end{aligned}
$$

In this present study, our objective is to further complement the previous studies conducted on the perturbed $(3+1)$ dimensional ZK equation by introducing the more general form by replacing the nonlinear term " $P P_{x}$ " with " $P^{k} P_{x}$ ". We now consider the generalized fractional form of this equation as

$$
\begin{aligned}
\mathcal{D}_{t}^{\gamma} P & +A P^{k} P_{x}+B P_{x x x}+C\left(P_{x y y}+P_{x z z}\right) \\
& +\mathcal{E} P_{x x x x x}=0, \quad 0<\gamma \leq 1, \quad t>0
\end{aligned}
$$

where $k$ is a positive number, $\gamma$ is the fractional-order and $\mathcal{E}$ is a smallness parameter. For this purpose, we have carefully proposed the sub-equation method of conformable type to find analytic closed-form solutions of Eq. (3). The solutions consist of the dark soliton, multi-soliton, kink-shape, solitary wave, bell-shaped solitons, and periodic solutions, which are all substantial in the field of mathematical physics. 
When $\gamma=1, k=1$, and $\mathcal{E}=0$, Eq. (3) reduces to the standard Zakharov?Kuznetsov (ZK) equation (see Eq. (1). For $\gamma=1, k=2$, and $\mathcal{E}=0$, Eq. (3) reduces to the modified $\mathrm{KdV}-\mathrm{ZK}$ equation developed for a plasma comprised of cool and hot electrons and a species of fluid ions [55]. The case when $k=1,2$ and 4 are considered in this present study. It is worth mentioning that the case when $\gamma=1$ and $k=1$ in Eq. (3) have been investigated by the following authors. Elwakil et al. in Ref. [56] have studied the electron-acoustic solitary waves in a magnetized collisionless plasma consisting of a cold electron fluid and non-thermal hot electrons obeying a non-thermal distribution, and stationary. Ali et al. in Ref. [57] have investigated the exact solutions by using a sine-cosine method and modified Kudryashov methods and constructed six Lie point symmetries and nonlocal conservation laws for this equation. Recently, in Ref. [58], using two methods, Lie symmetry analysis and generalized exponential rational function. The authors present new exact solutions through optimal systems of one-dimensional Lie subalgebras; some solitary waves depict single soliton, multisoliton, and annihilation profiles and six other closed-form solutions. To our knowledge, the case when $k=2$ and $k=4$ have not been studied before.

The rest of the paper is organized as follows: Section 2 gives a brief discussion of conformable derivatives which includes the definitions, basic properties, lemmas, and theorems. Section 3 presents the general idea of the sub-equation method. In Sec. 4, the application of sub-equation to timefractional perturbed $(3+1)$-dimensional ZK equation of conformable type is demonstrated. In Sec. 5, the graphical representation of some solutions is depicted in $3 \mathrm{D}$ for different fractional orders. Finally, Sec. 6 gives the conclusion.

\section{Preliminaries}

This section contains a brief discussion of conformable derivatives, which includes the definitions, basic properties, lemmas, and theorems. Most of the concepts presented in this section have been introduced in $[59,60]$

Definition 2.1. Let $P:[0, \infty) \rightarrow \mathbb{R}$. The conformable derivative of $P$ of order $\gamma$ is given by

$\mathcal{D}^{\gamma} P(t)=\lim _{\varepsilon \rightarrow 0} \frac{P\left(t+\varepsilon t^{1-\gamma}\right)-P(t)}{\varepsilon}, \forall t>0, \gamma \in(0,1)$.

Furthermore, If $P$ is $\gamma$-differentiable in some interval $(0, \zeta)$ where $\zeta>0$, and $\lim _{t \rightarrow 0} P^{(\gamma)}(t)$ exists. We define

$$
P^{(\gamma)}(0)=\lim _{t \rightarrow 0^{+}} P^{(\gamma)}(t)
$$

Lemma 2.2. [59] Let $\gamma \in(0,1]$ and $P, Q$ be $\gamma$-differentiable at a point $t>0$. Then

(i.) $\mathcal{D}^{\gamma}\left(\delta_{1} P+\delta_{2} Q\right)=\delta_{1} \mathcal{D}^{\gamma} P+\delta_{2} \mathcal{D}^{\gamma} Q, \forall \delta_{1}, \delta_{2} \in \mathbb{R}$.

(ii.) $\mathcal{D}^{\gamma}\left(t^{\sigma}\right)=\sigma t^{\sigma-\gamma}, \forall \sigma \in \mathbb{R}$.

(iii.) $\mathcal{D}^{\gamma}(P Q)=P \mathcal{D}^{\gamma} Q+Q \mathcal{D}^{\gamma} P$.

(iv.) $\mathcal{D}^{\gamma}\left(\frac{P}{Q}\right)=\frac{Q \mathcal{D}^{\gamma} P-P \mathcal{D}^{\gamma} Q}{Q^{2}}$, provided $Q \neq 0$.

(v.) $\mathcal{D}^{\gamma}(C)=0$, where $C$ is a constant.

Lemma 2.3. [59] Let $P$ be a differentiable and $\gamma$ differentiable function. Then

$$
\mathcal{D}^{\gamma} P(t)=t^{1-\gamma} \frac{\partial P(t)}{\partial t}
$$

Theorem 2.4. [61, 62] Let $P:(0, \infty) \rightarrow \mathcal{R}$ be a differentiable and $\gamma$-differentiable function. Let $Q$ be a differentiable function defined in the range of $P$. Then

$$
\begin{aligned}
\mathcal{D}_{t}^{\gamma}(P \circ Q)(t) & =t^{1-\gamma} Q(t)^{\gamma-1} \\
& \times\left. Q^{\prime}(t) \mathcal{D}_{t}^{\gamma}(P(\Phi))\right|_{\{\Phi=Q(t)\}}
\end{aligned}
$$

\section{Algorithm of the proposed method}

The main concept of the sub-equation method $[63,64]$ for solving FPDEs is presented below:

$$
F\left(P, P_{x}, P_{y}, P_{z}, P_{x x}, P_{y y}, P_{z z}, \cdots, \mathcal{D}_{t}^{\gamma} P\right)=0
$$

Here, $\mathcal{D}_{t}^{\gamma}$ is defined according to Sec. 2 with fractional order $\gamma$ and an unknown function $P=P(x, y, z, t)$.

Step 1: Let $P(x, y, z, t)=P(\xi), \xi=p x+q y+r z+s \frac{t^{\gamma}}{\gamma}$, where $p, q, r$ and $s$ are constants to be calculated respectively. With the help of the chain rule, we can transform Eq. (8) to an integer order nonlinear ODE given as

$$
G\left(P(\xi), P^{\prime}(\xi), P^{\prime \prime}(\xi), P^{\prime \prime \prime}(\xi), \cdots\right)=0 .
$$

Step 2: We suppose that Eq. (9) has the solution:

$$
P(\xi)=e_{0}+\sum_{j=1}^{N} e_{j} \vartheta^{j}(\xi), e_{N} \neq 0
$$


where $p, q, r, s$, and $e_{j}(j=0,1, \cdots, N)$, are constants to be obtained accordingly. Balancing the highest-order derivative and biggest nonlinear term in Eq. (9) provides value for the integer $N$. The function $\vartheta(\xi)$ satisfies the Riccati equation:

$$
\vartheta^{\prime}(\xi)=\sigma+\vartheta^{2}(\xi)
$$

where $\sigma$ is a constant and the solutions are given as

$$
\vartheta(\xi)= \begin{cases}-\sqrt{-\sigma} \tanh (\sqrt{-\sigma} \xi), & \sigma<0, \\ -\sqrt{-\sigma} \operatorname{coth}(\sqrt{-\sigma} \xi), & \sigma<0, \\ \sqrt{\sigma} \tan (\sqrt{\sigma} \xi), & \sigma>0, \\ -\sqrt{\sigma} \cot (\sqrt{\sigma} \xi), & \sigma>0, \\ -\frac{1}{\xi+\phi}, \quad \phi \quad \text { is a constant, } & \sigma=0 .\end{cases}
$$

Step 3: Substituting Eqs. (10) and (11) into Eq. (9), we have some polynomial in $\vartheta^{j}(\xi),(j=0,1,2 \cdots)$. Furthermore, setting the coefficients of $\vartheta^{j}(\xi)$ to zero, results set of nonlinear algebraic equations in $p, q, r, s$, and $e_{j}(j=0,1, \cdots, N)$.

Step 4: Finally, by solving the equations found in Step 3. Then, substitute these constants $p, q, r, s, \sigma$ and $e_{j}$ into Eq. (10) in addition to Eq. (12), we conclude the desirable solutions for Eq. (8) immediately.

\section{Sub-Equation Method to PZK Equations of Comformable Type}

Here, the application of the sub-equation method to the time-fractional perturbed $(3+1)$-dimensional ZK equation of conformable type is presented. Consider

$$
\mathcal{D}_{t}^{\gamma} P+A P^{k} P_{x}+B P_{x x x}+C\left(P_{x y y}+P_{x z z}\right)+\mathcal{E} P_{x x x x x}=0
$$

Application of chain rule on $P(x, y, z, t)=P(\xi), \xi=p x+q y+r z+s \frac{t^{\gamma}}{\gamma}$ reduces Eq. (13) to a nonlinear ODE given as

$$
\left(s+p A P^{k}\right) P^{\prime}+\left(p^{3} B+C\left(p q^{2}+p r^{2}\right)\right) P^{\prime \prime \prime}+p^{5} \mathcal{E} P^{\prime \prime \prime \prime \prime \prime}=0 .
$$

Balance principle to the terms $P^{k} P^{\prime}$ and $P^{\prime \prime \prime \prime \prime}$ yields $N=4 / k$. To obtain closed-form solutions, $N$ must be an integer; therefore, we choose $k=1,2$ and 4 .

\subsection{Solutions for $k=1$ :}

For $k=1$, we have $N=4$, then Eq. (10) gives

$$
P(\xi)=e_{0}+e_{1} \vartheta(\xi)+e_{2} \vartheta^{2}(\xi)+e_{3} \vartheta^{3}(\xi)+e_{4} \vartheta^{4}(\xi)
$$


By substituting Eqs. (11) and (15) into Eq. (14), then equating the coefficients of $\vartheta^{r}(\xi)$ to zero yields

$$
\begin{aligned}
\vartheta^{0}(\xi) & : A e_{0} e_{1} p \sigma+6 e_{3} \sigma^{3}\left(B p^{3}+C p q^{2}+C p r^{2}\right)+2 e_{1} \sigma^{2}\left(B p^{3}+C p q^{2}+C p r^{2}\right)+120 \mathcal{E} e_{3} p^{5} \sigma^{4} \\
& +16 \mathcal{E} e_{1} p^{5} \sigma^{3}+e_{1} s \sigma=0 \\
\vartheta^{1}(\xi) & : A e_{1}^{2} p \sigma+2 A e_{0} e_{2} p \sigma+24 e_{4} \sigma^{3}\left(B p^{3}+C p q^{2}+C p r^{2}\right)+16 e_{2} \sigma^{2}\left(B p^{3}+C p q^{2}+C p r^{2}\right) \\
& +960 \mathcal{E} e_{4} p^{5} \sigma^{4}+272 \mathcal{E} e_{2} p^{5} \sigma^{3}+2 e_{2} s \sigma=0 \\
\vartheta^{2}(\xi) & : 3 A e_{1} e_{2} p \sigma+3 A e_{0} e_{3} p \sigma+A e_{0} e_{1} p+60 e_{3} \sigma^{2}\left(B p^{3}+C p q^{2}+C p r^{2}\right) \\
& +8 e_{1} \sigma\left(B p^{3}+C p q^{2}+C p r^{2}\right)+1848 \mathcal{E} e_{3} p^{5} \sigma^{3}+136 \mathcal{E} e_{1} p^{5} \sigma^{2}+3 e_{3} s \sigma+e_{1} s=0, \\
\vartheta^{3}(\xi) & : 2 A e_{2}^{2} p \sigma+4 A e_{1} e_{3} p \sigma+4 A e_{0} e_{4} p \sigma+A e_{1}^{2} p+2 A e_{0} e_{2} p \\
& +152 e_{4} \sigma^{2}\left(B p^{3}+C p q^{2}+C p r^{2}\right)+40 e_{2} \sigma\left(B p^{3}+C p q^{2}+C p r^{2}\right) \\
& +7744 \mathcal{E} e_{4} p^{5} \sigma^{3}+1232 \mathcal{E} e_{2} p^{5} \sigma^{2}+4 e_{4} s \sigma+2 e_{2} s=0 \\
\vartheta^{4}(\xi) & : 5 A e_{2} e_{3} p \sigma+5 A e_{1} e_{4} p \sigma+3 A e_{1} e_{2} p+3 A e_{0} e_{3} p+114 e_{3} \sigma\left(B p^{3}+C p q^{2}+C p r^{2}\right) \\
& +6 e_{1}\left(B p^{3}+C p q^{2}+C p r^{2}\right)+5808 \mathcal{E} e_{3} p^{5} \sigma^{2}+240 \mathcal{E} e_{1} p^{5} \sigma+3 e_{3} s=0, \\
\vartheta^{5}(\xi) & : 3 A e_{3}^{2} p \sigma+6 A e_{2} e_{4} p \sigma+2 A e_{2}^{2} p+4 A e_{1} e_{3} p+4 A e_{0} e_{4} p+248 e_{4} \sigma\left(B p^{3}+C p q^{2}+C p r^{2}\right) \\
& +24 e_{2}\left(B p^{3}+C p q^{2}+C p r^{2}\right)+19264 \mathcal{E} e_{4} p^{5} \sigma^{2}+1680 \mathcal{E} e_{2} p^{5} \sigma+4 e_{4} s=0, \\
\vartheta^{6}(\xi) & : 7 A e_{3} e_{4} p \sigma+5 A e_{2} e_{3} p+5 A e_{1} e_{4} p+60 e_{3}\left(B p^{3}+C p q^{2}+C p r^{2}\right)+6600 \mathcal{E} e_{3} p^{5} \sigma+120 \mathcal{E} e_{1} p^{5}=0, \\
\vartheta^{7}(\xi) & : 4 A e_{4}^{2} p \sigma+3 A e_{3}^{2} p+6 A e_{2} e_{4} p+120 e_{4}\left(B p^{3}+C p q^{2}+C p r^{2}\right)+19200 \mathcal{E} e_{4} p^{5} \sigma+720 \mathcal{E} e_{2} p^{5}=0, \\
\vartheta^{8}(\xi) & : 7 A e_{3} e_{4} p+2520 \mathcal{E} e_{3} p^{5}=0, \\
\vartheta^{9}(\xi) & : 4 A e_{4}^{2} p+6720 \mathcal{E} e_{4} p^{5}=0 .
\end{aligned}
$$

With the aid of Mathematica, we solve the above equations as

\section{Case 1.}

$$
\begin{gathered}
e_{0}=e_{0}, \quad e_{1}=0, \quad e_{2}=-\frac{3360 \mathcal{E} p^{4} \sigma}{A}, \quad e_{3}=0, \quad e_{4}=-\frac{1680 \mathcal{E} p^{4}}{A}, \\
s=-A e_{0} p-1104 \mathcal{E} p^{5} \sigma^{2}, \quad r= \pm \sqrt{\frac{-B p^{2}-C q^{2}+52 \mathcal{E} p^{4} \sigma}{C}}
\end{gathered}
$$

By substituting Eq. (17) into Eq. (15) with solutions defined in Eq. (12), we have the required solutions of Eq. (13) as:

$$
\begin{array}{ll}
P_{1}=e_{0}+\frac{3360 \mathcal{E} p^{4} \sigma^{2}}{A} \tanh ^{2}\left(\sqrt{-\sigma}\left[p x+q y+r z+\frac{s}{\gamma} t^{\gamma}\right]\right)-\frac{1680 \mathcal{E} p^{4} \sigma^{2}}{A} \tanh ^{4}\left(\sqrt{-\sigma}\left[p x+q y+r z+\frac{s}{\gamma} t^{\gamma}\right]\right), & \sigma>0, \\
P_{2}=e_{0}+\frac{3360 \mathcal{E} p^{4} \sigma^{2}}{A} \operatorname{coth}^{2}\left(\sqrt{-\sigma}\left[p x+q y+r z+\frac{s}{\gamma} t^{\gamma}\right]\right)-\frac{1680 \mathcal{E} p^{4} \sigma^{2}}{A} \operatorname{coth}^{4}\left(\sqrt{-\sigma}\left[p x+q y+r z+\frac{s}{\gamma} t^{\gamma}\right]\right), & \sigma>0, \\
P_{3}=e_{0}-\frac{3360 \mathcal{E} p^{4} \sigma^{2}}{A} \tan ^{2}\left(\sqrt{\sigma}\left[p x+q y+r z+\frac{s}{\gamma} t^{\gamma}\right]\right)-\frac{1680 \mathcal{E} p^{4} \sigma^{2}}{A} \tan ^{4}\left(\sqrt{\sigma}\left[p x+q y+r z+\frac{s}{\gamma} t^{\gamma}\right]\right), & \sigma<0, \\
P_{4}=e_{0}-\frac{3360 \mathcal{E} p^{4} \sigma^{2}}{A} \cot ^{2}\left(\sqrt{\sigma}\left[p x+q y+r z+\frac{s}{\gamma} t^{\gamma}\right]\right)-\frac{1680 \mathcal{E} p^{4} \sigma^{2}}{A} \cot ^{4}\left(\sqrt{\sigma}\left[p x+q y+r z+\frac{s}{\gamma} t^{\gamma}\right]\right), & \sigma<0, \\
P_{5}=e_{0}-\frac{1680 \mathcal{E} p^{4}}{A\left(p x+q y \pm \sqrt{\frac{-B p^{2}-C q^{2}}{C}} z-\frac{A e_{0} p}{\gamma} t^{\gamma}+\phi\right)}, & \sigma=0,
\end{array}
$$

where $\phi$ is a constant, $s=-A e_{0} p-1104 \mathcal{E} p^{5} \sigma^{2}$ and $r= \pm \sqrt{\left(-B p^{2}-C q^{2}+52 \mathcal{E} p^{4} \sigma\right) / C}$. 


\section{Case 2.}

$$
\begin{aligned}
& e_{0}=e_{0}, \quad e_{1}=0, \quad e_{2}=\frac{1680 i\left(\sqrt{31} \mathcal{E} p^{4} \sigma+31 i \mathcal{E} p^{4} \sigma\right)}{31 A}, \quad e_{3}=0, \quad e_{4}=-\frac{1680 \mathcal{E} p^{4}}{A} \\
& s=\frac{1}{31}\left(-31 A e_{0} p-3720 \mathcal{E} p^{5} \sigma^{2}+1368 i \sqrt{31} \mathcal{E} p^{5} \sigma^{2}\right), \quad r= \pm \sqrt{-\frac{B p^{2}}{C}-\frac{26 \mathcal{E} p^{4} \sigma}{C}-\frac{78 i \mathcal{E} p^{4} \sigma}{\sqrt{31} C}-q^{2}}
\end{aligned}
$$

The required solutions for Eq. (13 are

$$
\begin{aligned}
& P_{6}=e_{0}-\frac{1680 i \sigma\left(\sqrt{31} \mathcal{E} p^{4} \sigma+31 i \mathcal{E} p^{4} \sigma\right)}{31 A} \tanh ^{2}\left(\sqrt{-\sigma}\left[p x+q y+r z+\frac{s}{\gamma} t^{\gamma}\right]\right) \\
& -\frac{1680 \mathcal{E} p^{4} \sigma^{2}}{A} \tanh ^{4}\left(\sqrt{-\sigma}\left[p x+q y+r z+\frac{s}{\gamma} t^{\gamma}\right]\right), \quad \sigma<0 \\
& P_{7}=e_{0}-\frac{1680 i \sigma\left(\sqrt{31} \mathcal{E} p^{4} \sigma+31 i \mathcal{E} p^{4} \sigma\right)}{31 A} \operatorname{coth}^{2}\left(\sqrt{-\sigma}\left[p x+q y+r z+\frac{s}{\gamma} t^{\gamma}\right]\right) \\
& -\frac{1680 \mathcal{E} p^{4} \sigma^{2}}{A} \operatorname{coth}^{4}\left(\sqrt{-\sigma}\left[p x+q y+r z+\frac{s}{\gamma} t^{\gamma}\right]\right), \quad \sigma<0 \\
& P_{8}=e_{0}+\frac{1680 i \sigma\left(\sqrt{31} \mathcal{E} p^{4} \sigma+31 i \mathcal{E} p^{4} \sigma\right)}{31 A} \tan ^{2}\left(\sqrt{\sigma}\left[p x+q y+r z+\frac{s}{\gamma} t^{\gamma}\right]\right) \\
& -\frac{1680 \mathcal{E} p^{4} \sigma^{2}}{A} \tan ^{4}\left(\sqrt{\sigma}\left[p x+q y+r z+\frac{s}{\gamma} t^{\gamma}\right]\right), \quad \sigma>0 \\
& P_{9}=e_{0}+\frac{1680 i \sigma\left(\sqrt{31} \mathcal{E} p^{4} \sigma+31 i \mathcal{E} p^{4} \sigma\right)}{31 A} \cot ^{2}\left(\sqrt{\sigma}\left[p x+q y+r z+\frac{s}{\gamma} t^{\gamma}\right]\right) \\
& -\frac{1680 \mathcal{E} p^{4} \sigma^{2}}{A} \cot ^{4}\left(\sqrt{\sigma}\left[p x+q y+r z+\frac{s}{\gamma} t^{\gamma}\right]\right) \\
& P_{10}=e_{0}-\frac{1680 \mathcal{E} p^{4}}{A\left(p x+q y \pm z \sqrt{-\frac{B p^{2}}{C}-q^{2}}-\frac{A e_{0} p}{\gamma} t \gamma+\phi\right)^{4}} \text {, } \\
& \sigma>0 \\
& \sigma=0
\end{aligned}
$$

where $s=\frac{1}{31}\left(-31 A e_{0} p-3720 \mathcal{E} p^{5} \sigma^{2}+1368 i \sqrt{31} \mathcal{E} p^{5} \sigma^{2}\right)$ and $r= \pm \sqrt{-\frac{B p^{2}}{C}-\frac{26 \mathcal{E} p^{4} \sigma}{C}-\frac{78 i \mathcal{E} p^{4} \sigma}{\sqrt{31} C}-q^{2}}$.

\section{Case 3.}

$$
\begin{aligned}
& e_{0}=e_{0}, \quad e_{1}=0, \quad e_{2}=-\frac{1680 i\left(\sqrt{31} \mathcal{E} p^{4} \sigma-31 i \mathcal{E} p^{4} \sigma\right)}{31 A}, \quad e_{3}=0, \quad e_{4}=-\frac{1680 \mathcal{E} p^{4}}{A} \\
& s=\frac{1}{31}\left(-31 A e_{0} p-1368 i \sqrt{31} \mathcal{E} p^{5} \sigma^{2}-3720 \mathcal{E} p^{5} \sigma^{2}\right), \quad r= \pm \sqrt{-\frac{B p^{2}}{C}+\frac{78 i \mathcal{E} p^{4} \sigma}{\sqrt{31} C}-\frac{26 \mathcal{E} p^{4} \sigma}{C}-q^{2}}
\end{aligned}
$$


The required solutions for Eq. (13 are

$$
\begin{aligned}
& P_{11}=e_{0}+\frac{1680 i \sigma\left(\sqrt{31} \mathcal{E} p^{4} \sigma-31 i \mathcal{E} p^{4} \sigma\right)}{31 A} \tanh ^{2}\left(\sqrt{-\sigma}\left[p x+q y+r z+\frac{s}{\gamma} t^{\gamma}\right]\right) \\
& -\frac{1680 \mathcal{E} p^{4} \sigma^{2}}{A} \tanh ^{4}\left(\sqrt{-\sigma}\left[p x+q y+r z+\frac{s}{\gamma} t^{\gamma}\right]\right), \quad \sigma<0 \\
& P_{12}=e_{0}+\frac{1680 i \sigma\left(\sqrt{31} \mathcal{E} p^{4} \sigma-31 i \mathcal{E} p^{4} \sigma\right)}{31 A} \operatorname{coth}^{2}\left(\sqrt{-\sigma}\left[p x+q y+r z+\frac{s}{\gamma} t^{\gamma}\right]\right) \\
& -\frac{1680 \mathcal{E} p^{4} \sigma^{2}}{A} \operatorname{coth}^{4}\left(\sqrt{-\sigma}\left[p x+q y+r z+\frac{s}{\gamma} t^{\gamma}\right]\right), \quad \sigma<0 \\
& P_{13}=e_{0}-\frac{1680 i \sigma\left(\sqrt{31} \mathcal{E} p^{4} \sigma-31 i \mathcal{E} p^{4} \sigma\right)}{31 A} \tan ^{2}\left(\sqrt{\sigma}\left[p x+q y+r z+\frac{s}{\gamma} t^{\gamma}\right]\right) \\
& -\frac{1680 \mathcal{E} p^{4} \sigma^{2}}{A} \tan ^{4}\left(\sqrt{\sigma}\left[p x+q y+r z+\frac{s}{\gamma} t^{\gamma}\right]\right), \quad \sigma>0 \\
& P_{14}=e_{0}-\frac{1680 i \sigma\left(\sqrt{31} \mathcal{E} p^{4} \sigma-31 i \mathcal{E} p^{4} \sigma\right)}{31 A} \cot ^{2}\left(\sqrt{\sigma}\left[p x+q y+r z+\frac{s}{\gamma} t^{\gamma}\right]\right) \\
& -\frac{1680 \mathcal{E} p^{4} \sigma^{2}}{A} \cot ^{4}\left(\sqrt{\sigma}\left[p x+q y+r z+\frac{s}{\gamma} t^{\gamma}\right]\right) \\
& P_{15}=e_{0}-\frac{1680 \mathcal{E} p^{4}}{A\left(p x+q y \pm z \sqrt{-\frac{B p^{2}}{C}-q^{2}}-\frac{A e_{0} p}{\gamma} t \gamma+\phi\right)^{4}}, \\
& \sigma>0 \\
& \sigma=0,
\end{aligned}
$$

where $s=\frac{1}{31}\left(-31 A e_{0} p-1368 i \sqrt{31} \mathcal{E} p^{5} \sigma^{2}-3720 \mathcal{E} p^{5} \sigma^{2}\right)$ and $r= \pm \sqrt{-\frac{B p^{2}}{C}+\frac{78 i \mathcal{E} p^{4} \sigma}{\sqrt{31} C}-\frac{26 \mathcal{E} p^{4} \sigma}{C}-q^{2}}$.

\subsection{Solutions for $k=2$ :}

When $k=2$, we have $N=2$, then Eq. (10) gives

$$
P(\xi)=e_{0}+e_{1} \vartheta(\xi)+e_{1} \vartheta^{2}(\xi)
$$

By substituting Eqs. (20) and (11) into Eq. (14), then equating the coefficients of $\vartheta^{r}(\xi)$ to zero yields

$$
\begin{aligned}
\vartheta^{0}(\xi) & : A e_{0}^{2} e_{1} p \sigma+2 e_{1} \sigma^{2}\left(B p^{3}+C p q^{2}+C p r^{2}\right)+16 \mathcal{E} e_{1} p^{5} \sigma^{3}+e_{1} s \sigma=0 \\
\vartheta^{1}(\xi) & : 2 A e_{0} e_{1}^{2} p \sigma+2 A e_{0}^{2} e_{2} p \sigma+16 e_{2} \sigma^{2}\left(B p^{3}+C p q^{2}+C p r^{2}\right)+272 \mathcal{E} e_{2} p^{5} \sigma^{3}+2 e_{2} s \sigma=0 \\
\vartheta^{2}(\xi) & : A e_{1}^{3} p \sigma+6 A e_{0} e_{1} e_{2} p \sigma+A e_{0}^{2} e_{1} p+8 e_{1} \sigma\left(B p^{3}+C p q^{2}+C p r^{2}\right)+136 \mathcal{E} e_{1} p^{5} \sigma^{2}+e_{1} s=0 \\
\vartheta^{3}(\xi) & : 4 A e_{0} e_{2}^{2} p \sigma+4 A e_{1}^{2} e_{2} p \sigma+2 A e_{0} e_{1}^{2} p+2 A e_{0}^{2} e_{2} p+40 e_{2} \sigma\left(B p^{3}+C p q^{2}+C p r^{2}\right) \\
& +1232 \mathcal{E} e_{2} p^{5} \sigma^{2}+2 e_{2} s=0 \\
\vartheta^{4}(\xi) & : 5 A e_{1} e_{2}^{2} p \sigma+A e_{1}^{3} p+6 A e_{0} e_{1} e_{2} p+6 e_{1}\left(B p^{3}+C p q^{2}+C p r^{2}\right)+240 \mathcal{E} e_{1} p^{5} \sigma=0 \\
\vartheta^{5}(\xi) & : 2 A e_{2}^{3} p \sigma+4 A e_{0} e_{2}^{2} p+4 A e_{1}^{2} e_{2} p+24 e_{2}\left(B p^{3}+C p q^{2}+C p r^{2}\right)+1680 \mathcal{E} e_{2} p^{5} \sigma=0 \\
\vartheta^{6}(\xi) & : 5 A e_{1} e_{2}^{2} p+120 \mathcal{E} e_{1} p^{5}=0 \\
\vartheta^{7}(\xi) & : 2 A e_{2}^{3} p+720 \mathcal{E} e_{2} p^{5}=0
\end{aligned}
$$

With the help of Mathematica, we solve the above equations as 


\section{Case 1.}

$$
\begin{aligned}
& e_{0}=e_{0}, \quad e_{1}=0, \quad e_{2}=-6 i p^{2} \sqrt{\frac{10 \mathcal{E}}{A}}, \quad s=-8 i \sqrt{10 A \mathcal{E}} e_{0} p^{3} \sigma-A e_{0}^{2} p+184 \mathcal{E} p^{5} \sigma^{2} \\
& r= \pm \sqrt{\frac{i \sqrt{10 A \mathcal{E}} e_{0} p^{2}-B p^{2}-C q^{2}-40 \mathcal{E} p^{4} \sigma}{C}}
\end{aligned}
$$

By substituting Eq. (22) into Eq. (20) and using the solutions defined in Eq. (12), we obtain the required solutions to Eq. (13) as:

$$
\begin{array}{ll}
P_{16}=e_{0}+6 i p^{2} \sigma \sqrt{\frac{10 \mathcal{E}}{A}} \tanh ^{2}\left(\sqrt{-\sigma}\left(p x+q y+r z+\frac{s}{\gamma} t^{\gamma}\right)\right), & \sigma<0, \\
P_{17}=e_{0}+6 i p^{2} \sigma \sqrt{\frac{10 \mathcal{E}}{A}} \operatorname{coth}^{2}\left(\sqrt{-\sigma}\left(p x+q y+r z+\frac{s}{\gamma} t^{\gamma}\right)\right), & \sigma<0, \\
P_{18}=e_{0}-6 i p^{2} \sigma \sqrt{\frac{10 \mathcal{E}}{A}} \tan ^{2}\left(\sqrt{\sigma}\left(p x+q y+r z+\frac{s}{\gamma} t^{\gamma}\right)\right), & \sigma>0, \\
P_{19}=e_{0}-6 i p^{2} \sigma \sqrt{\frac{10 \mathcal{E}}{A}} \cot ^{2}\left(\sqrt{\sigma}\left(p x+q y+r z+\frac{s}{\gamma} t^{\gamma}\right)\right), & \sigma>0, \\
P_{20}=e_{0}-\frac{6 i p^{2} \sqrt{10 \mathcal{E}}}{\sqrt{A}}\left(p x+q y \pm \sqrt{\frac{i \sqrt{10 A \mathcal{E}} e_{0} p^{2}-B p^{2}-C q^{2}}{C}} z-\frac{A e_{0}^{2} p}{\gamma} t^{\gamma}+\phi\right)^{2} & \sigma=0,
\end{array}
$$

where $s=-8 i \sqrt{10} \sqrt{A} \sqrt{\mathcal{E}} e_{0} p^{3} \sigma-A e_{0}^{2} p+184 \mathcal{E} p^{5} \sigma^{2}$ and $r= \pm \sqrt{\frac{i \sqrt{10 A \mathcal{E}} e_{0} p^{2}-B p^{2}-C q^{2}-40 \mathcal{E} p^{4} \sigma}{C}}$.

\section{Case 2.}

$$
\begin{aligned}
e_{0} & =e_{0}, \quad e_{1}=0, \quad e_{2}=6 i p^{2} \sqrt{\frac{10 \mathcal{E}}{A}}, \quad s=8 i \sqrt{10 A \mathcal{E}} e_{0} p^{3} \sigma-A e_{0}^{2} p+184 \mathcal{E} p^{5} \sigma^{2}, \\
r & = \pm \sqrt{\frac{-i \sqrt{10 A \mathcal{E}} e_{0} p^{2}-B p^{2}-C q^{2}-40 \mathcal{E} p^{4} \sigma}{C}}
\end{aligned}
$$

By substituting Eq. (23) into Eq. (20) and using the solutions defined in Eq. (12), we obtain the required solutions to Eq. (13) as:

$$
\begin{array}{ll}
P_{21}=e_{0}-6 i p^{2} \sigma \sqrt{\frac{10 \mathcal{E}}{A}} \tanh ^{2}\left(\sqrt{-\sigma}\left(p x+q y+r z+s \frac{t^{\gamma}}{\gamma}\right)\right), & \sigma<0, \\
P_{22}=e_{0}-6 i p^{2} \sigma \sqrt{\frac{10 \mathcal{E}}{A}} \operatorname{coth}^{2}\left(\sqrt{-\sigma}\left(p x+q y+r z+s \frac{t^{\gamma}}{\gamma}\right)\right), & \sigma<0, \\
P_{23}=e_{0}+6 i p^{2} \sigma \sqrt{\frac{10 \mathcal{E}}{A}} \tan ^{2}\left(\sqrt{\sigma}\left(p x+q y+r z+s \frac{t^{\gamma}}{\gamma}\right)\right), & \sigma>0, \\
P_{24}=e_{0}+6 i p^{2} \sigma \sqrt{\frac{10 \mathcal{E}}{A}} \cot ^{2}\left(\sqrt{\sigma}\left(p x+q y+r z+s \frac{t^{\gamma}}{\gamma}\right)\right), & \sigma>0, \\
P_{25}=e_{0}+\frac{6 i p^{2} \sqrt{10 \mathcal{E}}}{\sqrt{A}\left(p x+q y \pm \sqrt{\frac{-i \sqrt{10 A \mathcal{E}} e_{0} p^{2}-B p^{2}-C q^{2}}{C}} z-\frac{A e_{0}^{2} p}{\gamma} t^{\gamma}+\phi\right)^{2}}, \quad \sigma=0,
\end{array}
$$

where $s=8 i \sqrt{10 A \mathcal{E}} e_{0} p^{3} \sigma-A e_{0}^{2} p+184 \mathcal{E} p^{5} \sigma^{2}$ and $r= \pm \sqrt{\frac{-i \sqrt{10 A \mathcal{E}} e_{0} p^{2}-B p^{2}-C q^{2}-40 \mathcal{E} p^{4} \sigma}{C}}$. 


\subsection{Solutions for $k=4$ :}

When $k=4$, we have $N=1$, then Eq. (10) yields

$$
P(\xi)=e_{0}+e_{1} \vartheta(\xi)
$$

By substituting Eqs. (24) and (11) into Eq. (14), then equating the coefficients of $\vartheta^{r}(\xi)$ to zero gives

$$
\begin{aligned}
& \vartheta^{0}(\xi): A e_{0}^{4} e_{1} p \sigma+2 e_{1} \sigma^{2}\left(B p^{3}+C p q^{2}+C p r^{2}\right)+16 \mathcal{E} e_{1} p^{5} \sigma^{3}+e_{1} s \sigma=0, \\
& \vartheta^{1}(\xi): 4 A e_{0}^{3} e_{1}^{2} p \sigma=0, \\
& \vartheta^{2}(\xi): 6 A e_{0}^{2} e_{1}^{3} p \sigma+A e_{0}^{4} e_{1} p+8 e_{1} \sigma\left(B p^{3}+C p q^{2}+C p r^{2}\right)+136 \mathcal{E} e_{1} p^{5} \sigma^{2}+e_{1} s=0, \\
& \vartheta^{3}(\xi): 4 A e_{0} e_{1}^{4} p \sigma+4 A e_{0}^{3} e_{1}^{2} p=0, \\
& \vartheta^{4}(\xi): A e_{1}^{5} p \sigma+6 A e_{0}^{2} e_{1}^{3} p+6 e_{1}\left(B p^{3}+C p q^{2}+C p r^{2}\right)+240 \mathcal{E} e_{1} p^{5} \sigma=0, \\
& \vartheta^{5}(\xi): 4 A e_{0} e_{1}^{4} p=0, \\
& \vartheta^{6}(\xi): A e_{1}^{5} p+120 \mathcal{E} e_{1} p^{5}=0 .
\end{aligned}
$$

By utilizing Mathematica, we solve the above equations as

\section{Case 1:}

$$
e_{0}=e_{0}, \quad e_{1}= \pm(1+i) p \sqrt[4]{\frac{30 \mathcal{E}}{A}}, \quad s=24 \mathcal{E} p^{5} \sigma^{2}, \quad r= \pm \sqrt{\frac{-B p^{2}-C q^{2}-20 \mathcal{E} p^{4} \sigma}{C}} .
$$

By substituting Eq. (26) into Eq. (24) and using the solutions defined in Eq. (12), we obtain the required solutions to Eq. (13) as:

$$
\begin{array}{ll}
P_{26}= \pm(1+i) p \sqrt{-\sigma} \sqrt[4]{\frac{30 \mathcal{E}}{A}} \tanh \left(\sqrt{-\sigma}\left[p x+q y \pm \sqrt{\frac{-B p^{2}-C q^{2}-20 \mathcal{E} p^{4} \sigma}{C}} z+\frac{24 \mathcal{E} p^{5} \sigma^{2}}{\gamma} t^{\gamma}\right]\right), & \sigma<0, \\
P_{27}= \pm(1+i) p \sqrt{-\sigma} \sqrt[4]{\frac{30 \mathcal{E}}{A}} \operatorname{coth}\left(\sqrt{-\sigma}\left[p x+q y \pm \sqrt{\frac{-B p^{2}-C q^{2}-20 \mathcal{E} p^{4} \sigma}{C}} z+\frac{24 \mathcal{E} p^{5} \sigma^{2}}{\gamma} t^{\gamma}\right]\right), & \sigma<0, \\
P_{28}=\mp(1+i) p \sqrt{\sigma} \sqrt[4]{\frac{30 \mathcal{E}}{A}} \tan \left(\sqrt{\sigma}\left[p x+q y \pm \sqrt{\frac{-B p^{2}-C q^{2}-20 \mathcal{E} p^{4} \sigma}{C}} z+\frac{24 \mathcal{E} p^{5} \sigma^{2}}{\gamma} t^{\gamma}\right]\right), & \sigma>0, \\
P_{29}= \pm(1+i) p \sqrt{\sigma} \sqrt[4]{\frac{30 \mathcal{E}}{A}} \cot \left(\sqrt{\sigma}\left[p x+q y \pm \sqrt{\frac{-B p^{2}-C q^{2}-20 \mathcal{E} p^{4} \sigma}{C}} z+\frac{24 \mathcal{E} p^{5} \sigma^{2}}{\gamma} t^{\gamma}\right]\right), & \sigma>0, \\
P_{30}= \pm \frac{(1+i) p \sqrt[4]{30 \mathcal{E}}}{\sqrt[4]{A}\left(p x+q y \pm \sqrt{\frac{-B p^{2}}{C}-q^{2}} z+\phi\right)}, & \sigma=0 .
\end{array}
$$

\section{Case 2:}

$$
e_{0}=e_{0}, \quad e_{1}= \pm(-1+i) p \sqrt[4]{\frac{30 \mathcal{E}}{A}}, \quad s=24 \mathcal{E} p^{5} \sigma^{2}, \quad r= \pm \sqrt{\frac{-B p^{2}-C q^{2}-20 \mathcal{E} p^{4} \sigma}{C}}
$$

By substituting Eq. (27) into Eq. (24) and using the solutions defined in Eq. (12), we obtain the required solutions to Eq. (13) 
as:

$$
\begin{aligned}
& P_{31}= \pm(-1+i) p \sqrt{-\sigma} \sqrt[4]{\frac{30 \mathcal{E}}{A}} \tanh \left(\sqrt{-\sigma}\left[p x+q y \pm \sqrt{\frac{-B p^{2}-C q^{2}-20 \mathcal{E} p^{4} \sigma}{C}} z+\frac{24 \mathcal{E} p^{5} \sigma^{2}}{\gamma} t^{\gamma}\right]\right), \quad \sigma<0 \\
& P_{32}= \pm(-1+i) p \sqrt{-\sigma} \sqrt[4]{\frac{30 \mathcal{E}}{A}} \operatorname{coth}\left(\sqrt{-\sigma}\left[p x+q y \pm \sqrt{\frac{-B p^{2}-C q^{2}-20 \mathcal{E} p^{4} \sigma}{C}} z+\frac{24 \mathcal{E} p^{5} \sigma^{2}}{\gamma} t^{\gamma}\right]\right), \quad \sigma<0 \\
& P_{33}=\mp(-1+i) p \sqrt{\sigma} \sqrt[4]{\frac{30 \mathcal{E}}{A}} \tan \left(\sqrt{\sigma}\left[p x+q y \pm \sqrt{\frac{-B p^{2}-C q^{2}-20 \mathcal{E} p^{4} \sigma}{C}} z+\frac{24 \mathcal{E} p^{5} \sigma^{2}}{\gamma} t^{\gamma}\right]\right), \quad \sigma>0 \text {, } \\
& P_{34}= \pm(-1+i) p \sqrt{\sigma} \sqrt[4]{\frac{30 \mathcal{E}}{A}} \cot \left(\sqrt{\sigma}\left[p x+q y \pm \sqrt{\frac{-B p^{2}-C q^{2}-20 \mathcal{E} p^{4} \sigma}{C}} z+\frac{24 \mathcal{E} p^{5} \sigma^{2}}{\gamma} t^{\gamma}\right]\right), \quad \sigma>0 \\
& P_{35}= \pm \frac{(-1+i) p \sqrt[4]{30 \mathcal{E}}}{\sqrt[4]{A}\left(p x+q y \pm \sqrt{\frac{-B p^{2}}{C}-q^{2}} z+\phi\right)},
\end{aligned}
$$

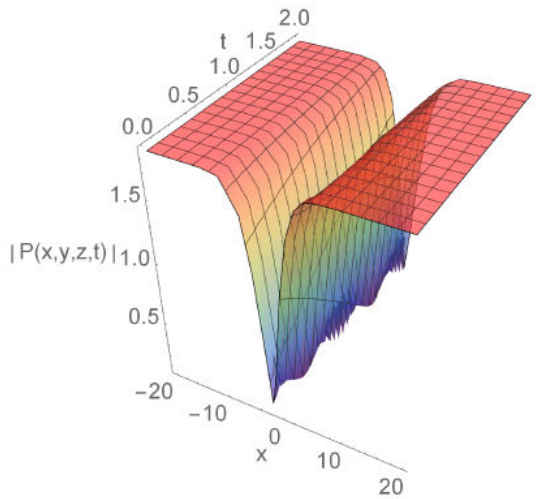

(a) $\gamma=1$

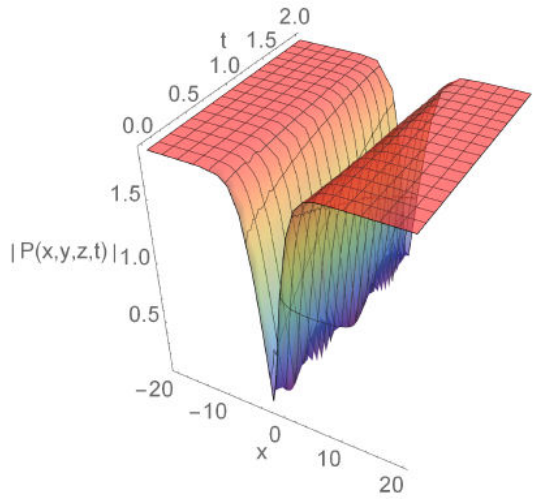

(b) $\gamma=0.75$

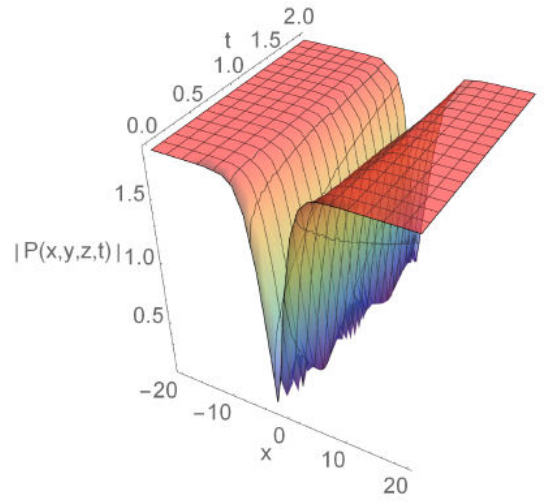

(c) $\gamma=0.5$

FIgURE 1. The plots of $P_{1}$ solution for $A=1, B=2, C=-20, y=z=2, \mathcal{E}=0.5, \sigma=-1, e_{0}=0.5, p=0.2$ and $q=0.2$.

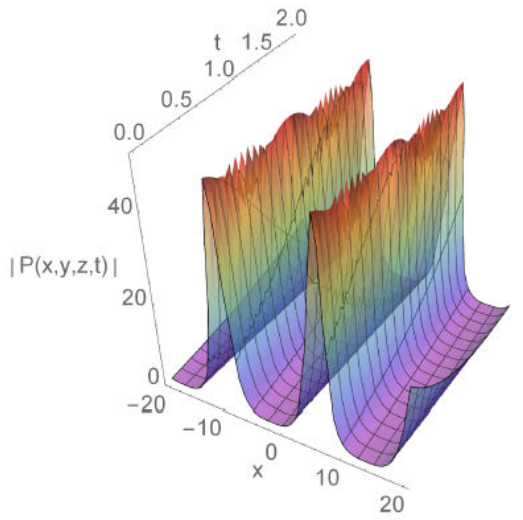

(a) $\gamma=1$

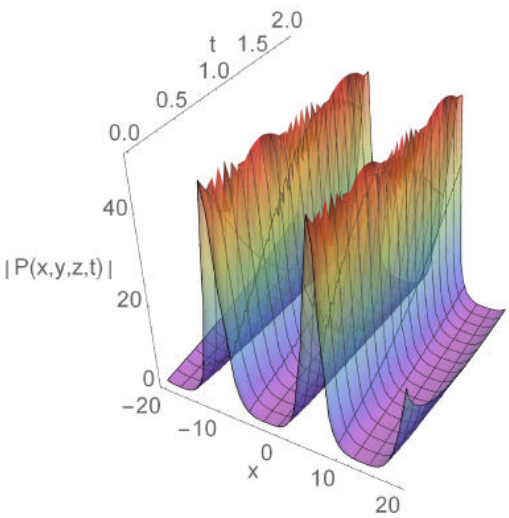

(b) $\gamma=0.75$

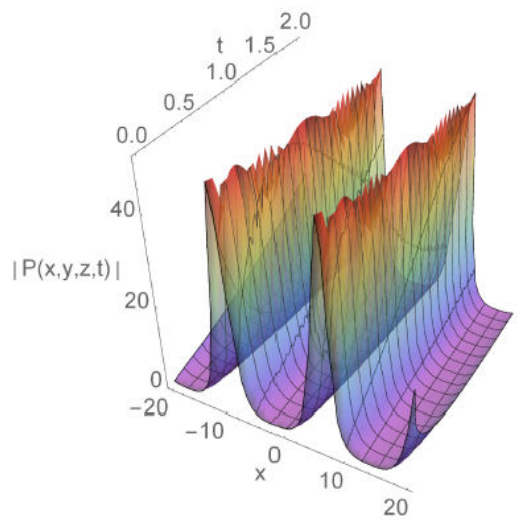

(c) $\gamma=0.5$

FIgURE 2. The plots of $P_{3}$ solution for $A=1, B=2, C=-20, y=z=2, \mathcal{E}=0.5, \sigma=1, e_{0}=0.5, p=0.2$ and $q=0.2$. 


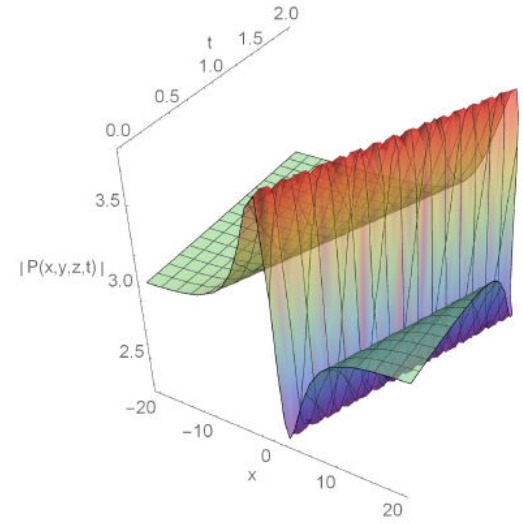

(a) $\gamma=1$

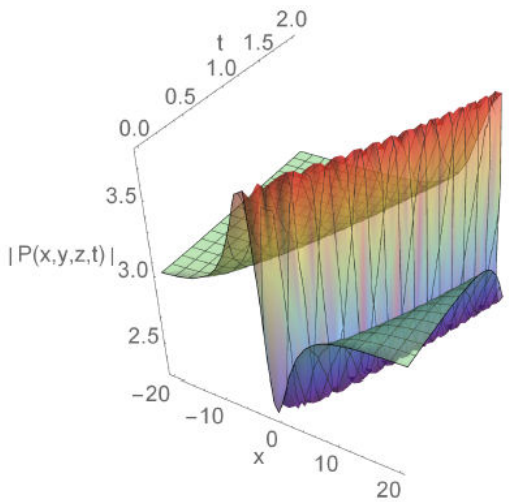

(b) $\gamma=0.75$

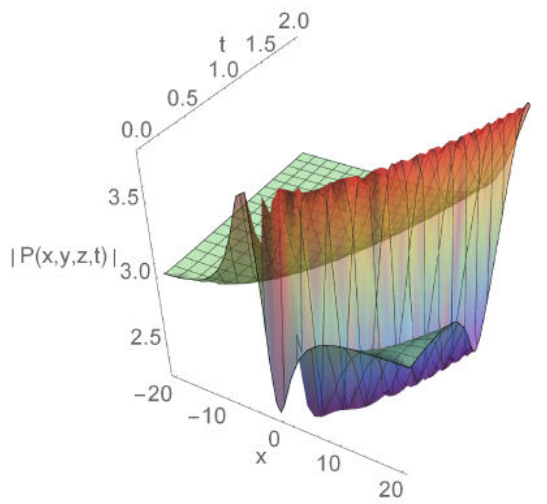

(c) $\gamma=0.5$

FIGURE 3. The plots of $P_{20}$ solution for $A=1, B=2, C=-1, y=z=2, \mathcal{E}=0.5, \phi=1, e_{0}=3, p=0.2$ and $q=0.2$.

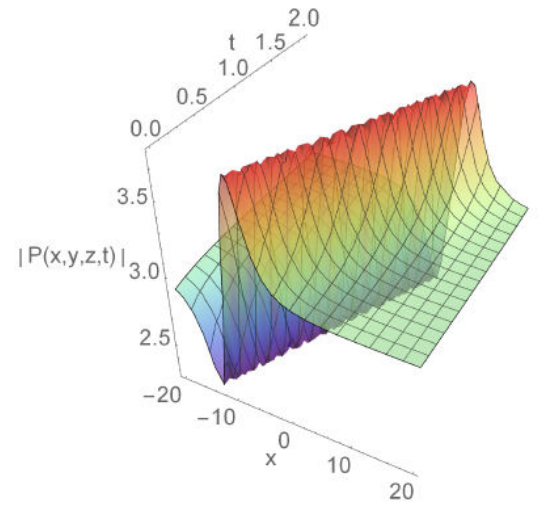

(a) $\gamma=1$

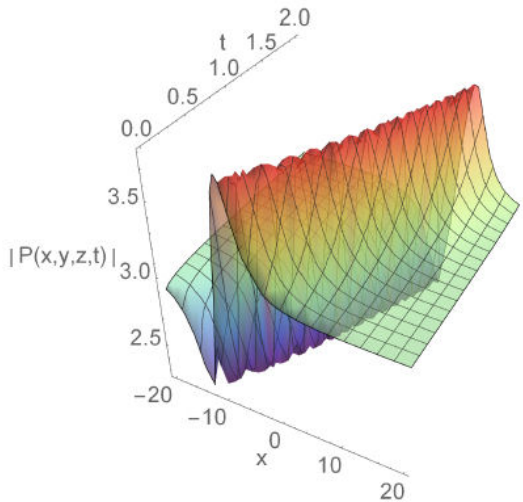

(b) $\gamma=0.75$

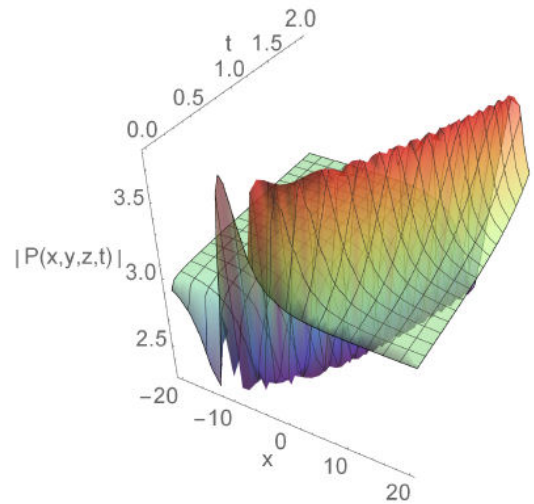

(c) $\gamma=0.5$

FIGURE 4. The plots of $P_{25}$ solution for $A=1, B=2, C=-1, y=z=2, \mathcal{E}=0.5, \phi=1, e_{0}=3, p=0.2$ and $q=0.2$.

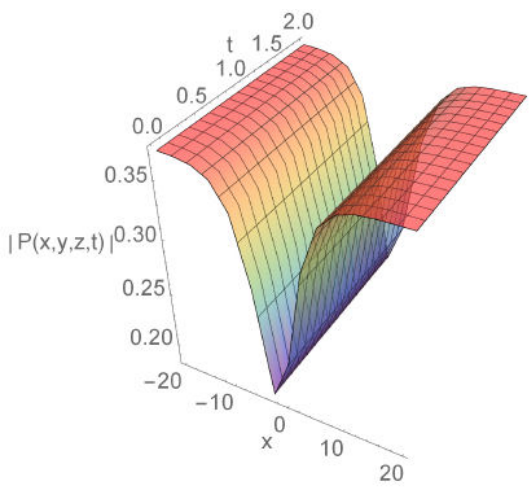

(a) $\gamma=1$

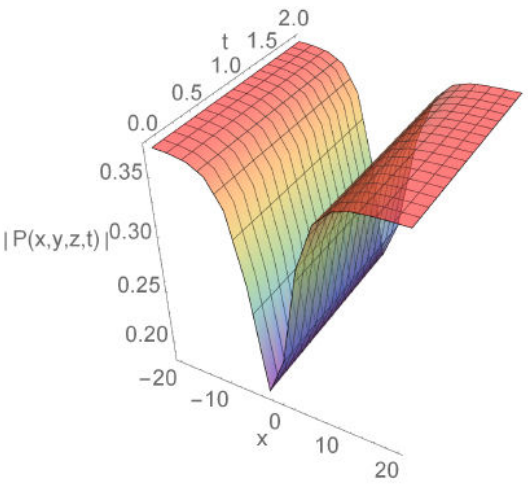

(b) $\gamma=0.75$

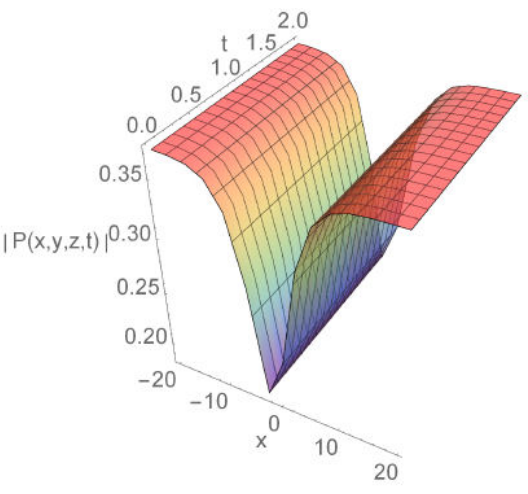

(c) $\gamma=0.5$

FIGURE 5. The plots of $P_{26}$ solution for $A=1, B=2, C=10, y=z=2, \mathcal{E}=0.1, \sigma=-1, e_{0}=3, p=0.2$ and $q=0.2$. 


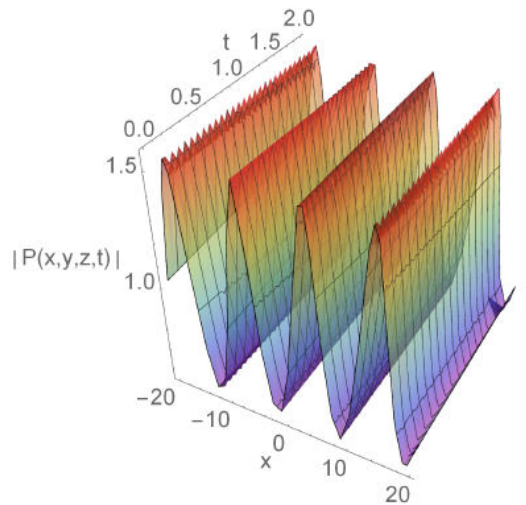

(a) $\gamma=1$

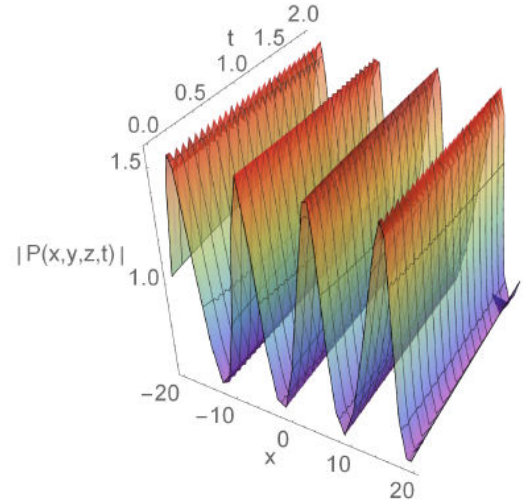

(b) $\gamma=0.75$

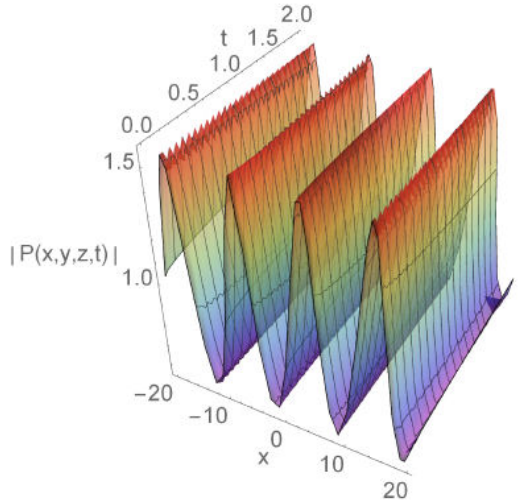

(c) $\gamma=0.5$

FIGURE 6. The plots of $P_{28}$ solution for $A=1, B=2, C=10, y=z=2, \mathcal{E}=0.8, \sigma=1, e_{0}=3, p=0.3$ and $q=0.3$.

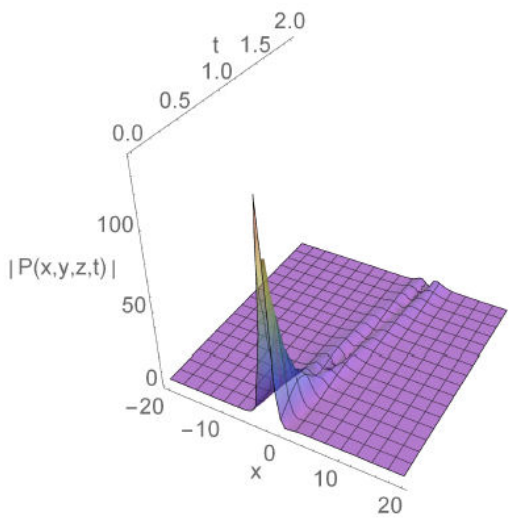

(a) $\mathcal{E}=0.1$

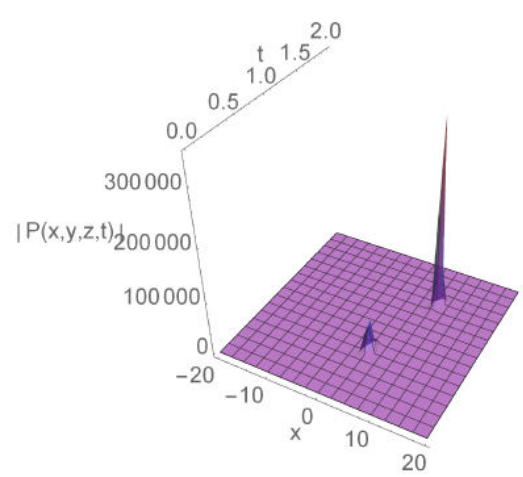

(b) $\mathcal{E}=0.5$

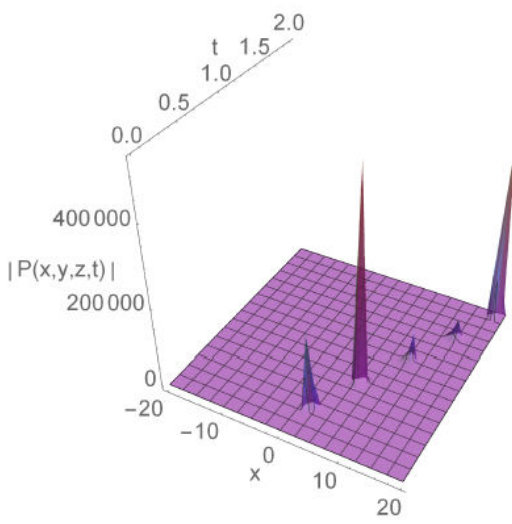

(c) $\mathcal{E}=1$

Figure 7. The effect of the parameter $\mathcal{E}$ on solution profile of $P_{11}$ for $\gamma=1, A=1, B=2, C=10, y=z=2, \sigma=-1, e_{0}=3, p=$ 0.5 and $q=0.5$.

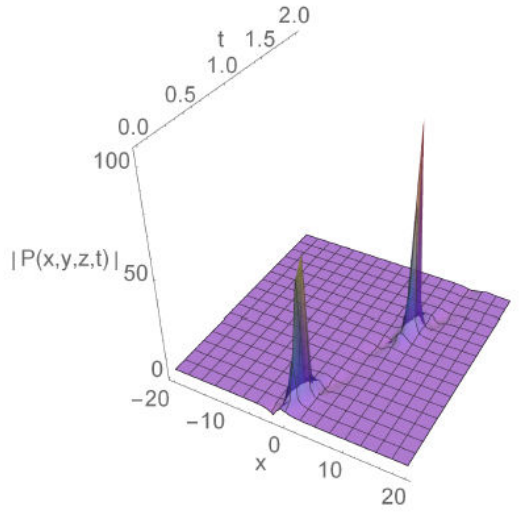

(a) $\mathcal{E}=0.1$

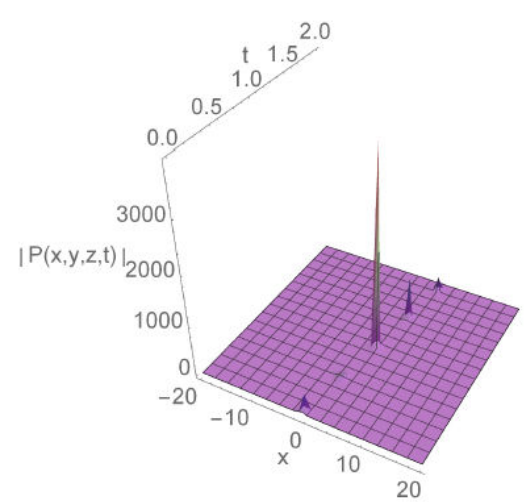

(b) $\mathcal{E}=0.5$

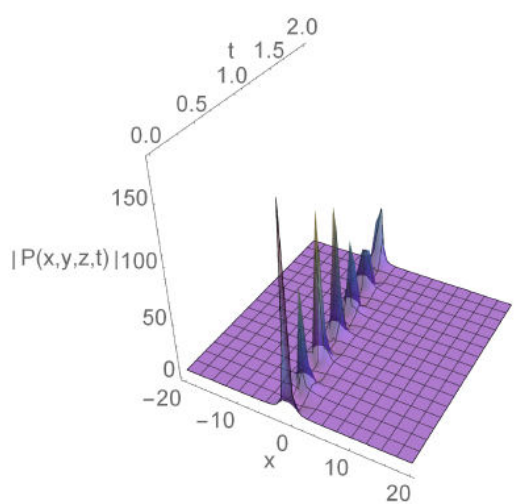

(c) $\mathcal{E}=1$

FIGURE 8. The effect of the parameter $\mathcal{E}$ on solution profile of $P_{16}$ for $\gamma=1, A=1, B=2, C=-5, y=z=2, \sigma=-1, e_{0}=3, p=$ 0.5 and $q=0.5$. 


\section{Graphical representation of some solutions}

The absolute behavior in 3D plots with integer and fractional order respectively $\gamma=1, \gamma=0.75$, and 0.5 are presented for some solutions in Fig. 1-8. These plots reveal different structures such as the dark soliton, multi-soliton, solitary wave, bell-shaped soliton, periodic and kink-type solutions which give the readers a better vision of the behavior of these solutions and captured some physical features of the considered model. Furthermore, Fig. 7 and 8 display the effect of adding a new perturbation term on the profile of the solution.

\section{Conclusion}

In this paper, we introduced the generalized time-fractional perturbed $(3+1)$ Zakharov-Kuznetsov (PZK) equations which describe the nonlinear dust-ion-acoustic waves in the magnetized two-ion-temperature dusty plasmas. We investi- gate the exact solutions by the use the of sub-equation method in the conformable sense. The use of conformable derivative in this study gives flexibility when applying to the proposed model and satisfies the power rule, product rule, quotient rule, integration by parts, chain rule, linearity, and the derivatives with constant is zero. The newly obtained solutions by the proposed method are, respectively, the dark soliton multisoliton, kink-shape, solitary wave, periodic and bell-shaped soliton solutions that are significant in the field of mathematical physics. Graphical representation (see Fig. 1 to 8) of obtained solutions are plotted in 3D for particular values of parameters. Figures 7 and 8, demonstrate the effect of adding a higher-order dispersion term "E $\mathcal{E} P_{x x x x x}$ " to Eq. (1). The performance of this method is reliable and effective and the obtained results are in a more general form. Finally, through Mathematica, we have authenticated the obtained solutions by substituting them back into the proposed equation.
1. L. Akinyemi, M. Senol, M. Mirzazadeh, and M. Eslami, Optical solitons for weakly nonlocal Schrödinger equation with parabolic law nonlinearity and external potential, Optik 230 (2021) 1, https://doi.org/10.1016/j.ijleo. 2021.166281

2. L. Akinyemi, M. Senol, and S. N. Husen, Modified homotopy methods for generalized fractional perturbed Zakharov-Kuznetsov equation in dusty plasma. Adv. Differ. Equ. 2021 (2021) 1, https://doi.org/10.1186/ s13662-020-03208-5

3. M. Mirzazadeh, L. Akinyemi, M. Senol, and K. Hosseini, A variety of solitons to the sixth-order dispersive $(3+1)$ dimensional nonlinear time-fractional Schrodinger equation with cubic-quintic-septic nonlinearities, Optik (2021) 166318, https://doi.org/10.1016/j.ijleo.2021. 166318

4. M.J. Ablowitz, Nonlinear Dispersive Waves: Asymptotic Analysis and Solitons, Cambridge University Press: Cambridge, (2011)

5. I. Owusu-Mensah, L. Akinyemi, B. Oduro, and O. S. Iyiola, A fractional order approach to modeling and simulations of the novel COVID-19. Adv. Differ. Equ. 2020 (2020) 1, https: //doi.org/10.1186/s13662-020-03141-7

6. A. Biswas et al., Optical solitons having weak non-local nonlinearity by two integration schemes, Optik. 164 (2018) 380.

7. Z. Yan, Abundant families of Jacobi elliptic function solutions of the $(2+1)$-dimensional integrable Davey-Stewartson-type equation via a new method. Chaos Soliton Fract. 18 (2003) 299, https://doi.org/10.1016/S0960-0779(02) 00653-7

8. G. Adomian, Solving Frontier Problems of Physics: The Decomposition Method. Kluwer, (1994).

9. A. M. Wazwaz, The sine-cosine method for obtaining solutions with compact and noncompact structures. Appl. Math. Comput.
159 (2004) 559, https://doi.org/10.1016/j.amc. 2003.08 .136

10. A. M. Wazwaz, A sine-cosine method for handling nonlinear wave equations. Math. Comput. Model. 40 (2004) 499, https://doi.org/10.1016/j.mcm.2003.12.010

11. S. El-Ganaini, Solutions of some class of nonlinear PDEs in mathematical physics. J. Egypt. Math. Soc. 24 (2016) 214, http://dx.doi.org/10.1016/j.joems.2015. 02.005 .

12. S. I. A. El-Ganaini, Traveling wave solutions to the generalized Pochhammer-Chree (PC) equations using the first integral method Math. Probl. Eng. 2011 (2011) 1, doi $10.1155 /$ 2011/629760

13. S. Das, Analytical solution of a fractional diffusion equation by variational iteration method. Comput. Math. Appl. 57 (2009) 483, https://doi.org/10.1016/j.camwa. 2008.09 .045

14. J. H. He, Approximate analytical solution for seepage flow with fractional derivatives in porous media. Comput. Meth. Appl. Mech. Eng. 167 (1998) 57, https: // doi.org/10.1016/ S0045-7825(98)00108-X

15. E. Fan, Extended tanh-function method and its applications to nonlinear equations. Phys. Lett. A 277 (2000) 212, https: //doi.org/10.1016/S0375-9601(00)00725-8

16. S. A. El-Wakil and M. A. Abdou, New exact travelling wave solutions using modified extended tanh-function method. Chaos Soliton Fract. 31 (2007) 840, https://doi.org/ $10.1016 / j . c h a o s .2005 .10 .032$

17. L. Akinyemi, q-Homotopy analysis method for solving the seventh-order time-fractional Lax's Korteweg-de Vries and Sawada-Kotera equations. Comp. Appl. Math. 38 (2019) 1, https://doi.org/10.1007/s40314-019-0977-3.

18. L. Akinyemi, O.S. Iyiola, and U. Akpan, Iterative methods for solving fourth- and sixth order time-fractional Cahn-Hillard 
equation. Math. Meth. Appl. Sci. 43 (2020) 4050. https: //doi.org/10.1002/mma.6173

19. M.A. El-Tawil and S.N. Huseen, The Q-homotopy analysis method (q-HAM). Int. J. Appl. Math. Mech. 8 (2012) 51.

20. J.H., He and X.H. Wu, Exp-function method for nonlinear wave equations. Chaos Solitons Fract. 30 (2006) 700, https : //doi.org/10.1016/j.chaos.2006.03.020

21. E. Yusufoglu, New solitary solutions for the MBBM equations using Exp-function method. Phys. Letters A 372 (2008) 442, https://doi.org/10.1016/j.physleta.2007. 07.062 .

22. S. Zhang, Application of Exp-function method to highdimensional nonlinear evolution equation. Chaos, Solitons Fract. 38 (2008) 270, DOI: $10.1016 / j . c h a o s .2006$. 11.014

23. L. Akinyemi and S.N. Huseen, A powerful approach to study the new modified coupled Korteweg-de Vries system. Math. Comput. Simul. 177 (2020) 556, https://doi.org/10. $1016 / j . m a t c o m .2020 .05 .021$

24. L. Akinyemi and O.S. Iyiola, A reliable technique to study nonlinear time-fractional coupled Korteweg-de Vries equations. Adv. Differ. Equ. 2020(169) (2020) 1, https://doi.org/ $10.1186 / \mathrm{s} 13662-020-02625-\mathrm{w}$

25. D. Kumara, J. Singha, and D. Baleanu, A new analysis for fractional model of regularized long-wave equation arising in ion acoustic plasma waves. Math. Meth. Appl. Sci. 40 (2017) 5642. https://doi.org/10.1002/mma.4414

26. W. Malflietand W. Hereman, The tanh method: I. Exact solutions of nonlinear evolution and wave equations. Phys. Scripta 54 (1996) 563, https://doi.org/10.1088/ 0031-8949/54/6/003.

27. A. M. Wazwaz, The tanh method for travelling wave solutions of nonlinear equations. Appl. Math. Comput. 154 (2004) 713, https://doi.org/10.1016/S0096-3003(03) 00745-8

28. J.H. He, Homotopy perturbation technique. Comput. Meth. Appl. Mech. Eng. 178 (1999) 257, http://dx.doi.org/ $10.1016 / \mathrm{S} 0045-7825(99) 00018-3$

29. M. Wang, X. Li, and J. Zhang, The $\frac{G^{\prime}}{G}$-expansion method and travelling wave solutions of nonlinear evolution equations in mathematical physics. Phys. Letters A 372 (2008) 417, https://doi.org/10.1016/j. physleta.2007.07.051

30. A. Bekir, Application of the $\frac{G^{\prime}}{G}$-expansion method for nonlinear evolution equations. Phys. Letters A 372 (2008) 3400, https://doi.org/10.1016/j.physleta.2008. 01.057 .

31. Y. Keskin and G. Oturanc, Reduced differential transform method: a new approach to fractional partial differential equations. Nonlinear Sci. Lett. A 1 (2010) 61.

32. L. Akinyemi, A fractional analysis of Noyes-Field model for the nonlinear Belousov-Zhabotinsky reaction. Comp. Appl. Math. 39 (2020) 1, https://doi.org/10.1007/ s40314-020-01212-9

33. E.G. Fan and Q.H. Zhang, A note on the homogeneous balance method. Phys. Lett. A 246 (1998) 403, https : / / doi .org/ $10.1016 / \mathrm{S} 0375-9601$ (98)00547-7
34. M. L. Wang, Exact solutions for a compound KdV-Burgers equation. Phys. Lett. A 213 (1996) 279, https://doi.org/ 10.1016/0375-9601(96)00103-X

35. V.O. Vakhnenko, E.J. Parkes, and A.J. Morrison, A Bäcklund transformation and the inverse scattering transform method for the generalised Vakhnenko equation. Chaos Soliton Fract. 17 (2003) 683, https://doi.org/10.1016/ S0960-0779(02)00483-6

36. L. Akinyemi and O.S. Iyiola, Exact and approximate solutions of time-fractional models arising from physics via Shehu transform. Math. Meth. Appl. Sci. (2020) 1, https://doi.org/ $10.1002 / \mathrm{mma} .6484$

37. S.J. Liao, On the homotopy analysis method for nonlinear problems. Appl. Math. Comput. 147 (2004) 499, https : //doi. org/10.1016/S0096-3003(02)00790-7.

38. S. J. Liao, An approximate solution technique not depending on small parameters: a special example. Intern. J. Non-linear Mech. 30 (1995) 371, https://doi.org/10. 1016/0020-7462(94)00054-E

39. O. Tasbozan, Y. Çenesiz, and A. Kurt, New solutions for conformable fractional Boussinesq and combined KdV-mKdV equations using Jacobi elliptic function expansion method. The European Physical Journal Plus 131 (2016) 1, https:// doi.org/10.1140/epjp/i2016-16244-x

40. O. Tasbozan, A. Kurt, and A. Tozar, New optical solutions of complex Ginzburg-Landau equation arising in semiconductor lasers. Appl. Phys. B 125 (2019) 1, https: / / doi.org/10. 1007/s00340-019-7217-9

41. M. Alquran, K. Al-Khaled, and J. Chattopadhyay, Analytical solutions of fractional population diffusion model: residual power series. Nonlinear Studies 22 (2015) 31.

42. M. Senol, O.S. Iyiola, H. Daei Kasmaei and L. Akinyemi, Efficient analytical techniques for solving time-fractional nonlinear coupled Jaulent-Miodek system with energy-dependent Schrödinger potential. Adv. Differ. Equ. 2019 (2019) 1, https://doi.org/10.1186/s13662-019-2397-5

43. M. Senol, Analytical and approximate solutions of $(2+1)$ dimensional time-fractional Burgers-Kadomtsev-Petviashvili equation. Commun. Theor. Phys. 72 (2020) 1, https:// doi.org/10.1088/1572-9494/ab7707

44. M. Senol and I.T. Dolapci, On the Perturbation-Iteration Algorithm for fractional differential equations. Journal of King Saud University-Science 28 (2016) 69, https://doi.org/10. $1016 / j \cdot j k s u s .2015 .09 .005$.

45. M. Senol, S. Atpinar, Z. Zararsiz, S. Salahshour, and A. Ahmadian, Approximate solution of time-fractional fuzzy partial differential equations. Comput. Appl. Math. 38 (2019) 1, https://doi.org/10.1007/s40314-019-0796-6

46. H. M. Srivastava et al., Traveling wave solutions to nonlinear directional couplers by modified Kudryashov method. Physica Scripta 95 (2020) 075217, https : / doi.org/10.1088/ $1402-4896 / a b 95 a f$

47. H. Rezazadeh, New solitons solutions of the complex Ginzburg-Landau equation with Kerr law nonlinearity. $O p$ tik, 167 (2018) 218, https://doi.org/10.1016/j. ijleo.2018.04.026 
48. H. Rezazadeh, M. Inc and D. Baleanu, New Solitary Wave Solutions for Variants of $(3+1)$-Dimensional Wazwaz-BenjaminBona-Mahony Equations. Frontiers in Physics, 8 (2020) 1, https://doi.org/10.3389/fphy.2020.00332

49. J. Vahidi et al., New solitary wave solutions to the coupled Maccari's system. Results in Physics, 21 (2021) 103801, https://doi.org/10.1016/j.rinp.2020. 103801 .

50. E. A. Az-Zo'bi et al., Abundant closed-form solitons for timefractional integro-differential equation in fluid dynamics. $O p t$ Quant Electron 53 (2021) 132. https://doi.org/10. 1007/s11082-021-02782-6

51. A.R. Seadawy and D. Lu, Ion acoustic solitary wave solutions of three-dimensional nonlinear extended Zakharov-Kuznetsov dynamical equation in a magnetized two-ion-temperature dusty plasma. Results Phys. 6 (2016) 590, https://doi.org/ 10.1016/j.rinp.2016.08.023

52. D. Lu, A.R. Seadawy, M. Arshad, and J. Wang, New solitary wave solutions of $(3+1)$-dimensional nonlinear extended Zakharov-Kuznetsov and modified KdV-ZakharovKuznetsov equations and their applications. Results Phys. 7 (2017) 899, https://doi.org/10.1016/j.rinp. 2017.02 .002

53. H. Zhen, B. Tian, Y. Wang, W. Sun, and L. Liu, Soliton solutions and chaotic motion of the extended Zakharov-Kuznetsov equations in a magnetized two-ion-temperature dusty plasma. Phys Plasma 21 (2014) 073709, https://doi.org/10. $1063 / 1.4885380$

54. S. Kumar and D. Kumar, Solitary wave solutions of $(3+$ 1)-dimensional extended Zakharov-Kuznetsov equation by Lie symmetry approach. Comput. Math. Appl. 77 (2019) 2096, https://doi.org/10.1016/j.camwa.2018. 12.009

55. R.L. Mace and M.A. Hellberg, The Korteweg-de VriesZakharov-Kuznetsov equation for electron-acoustic waves. Physics of Plasmas. 8 (2001) 2649, https://doi.org/ $10.1063 / 1.1363665$
56. S.A. Elwakil, E.K. El-Shewy, and H.G. Abdelwahed, Solution of the perturbed Zakharov-Kuznetsov (ZK) equation describing electron-acoustic solitary waves in a magnetized plasma. Chin. J. Phys. 49 (2011) 732.

57. M.N. Ali, A.R. Seadawy, and S.M. Husnine, Lie point symmetries exact solutions and conservation laws of perturbed Zakharov-Kuznetsov equation with higher-order dispersion term. Mod. Phys. Lett. A 34 (2019) 1950027, https : / / doi. org/10.1142/S0217732319500275

58. D. Kumar and S. Kumar, Solitary wave solutions of PZK equation using lie point symmetries. Eur. Phys. J. Plus 135 (2020) 1, https://doi.org/10.1140/epjp/ s13360-020-00218-w

59. R. Khalil, M. Al Horani, A. Yousef, and M. Sababheh, A new definition of fractional derivative. J. Comput. Appl. Math. 264 (2014) 65, https://doi.org/10.1016/j.cam. 2014.01 .002

60. T. Abdeljawad, On conformable fractional calculus. J. Comput. Appl. Math. 279 (2015) 57, https://doi.org/10. $1016 / j . c a m .2014 .10 .016$

61. A. Atangana, D. Baleanu, and A. Alsaedi, New properties of conformable derivative. Open Math. 13 (2015) 889, DOI: 10.1515/math-2015-0081

62. M. Eslami, and H. Rezazadeh, The first integral method for Wu-Zhang system with conformable time-fractional derivative. Calcolo 53 (2016) 475.

63. S. Zhang, and H.Q. Zhang, Fractional sub-equation method and its applications to nonlinear fractional PDEs. Phys. Lett. A 375 (2011) 1069, https://doi.org/10.1016/j. physleta.2011.01.029

64. L. Akinyemi, M. Senol, and O.S. Iyiola, Exact solutions of the generalized multidimensional mathematical physics models via sub-equation method. Math. Comput. Simul. 182 (2021) 211. https://doi.org/10.1016/j.matcom.2020. 10.017 